\title{
Theoretical Considerations for a Geosynchronous, Earth-Based Gravity Wave Interferometer
}

\author{
William P. Griffin \\ Department of Physics \\ University of Northern lowa \\ Cedar Falls, lowa 50614-0150 USA
}

Received: March 21, 2005 Accepted: May 6, 2005

\begin{abstract}
We investigated theoretical considerations in the design of an Earth-based laser interferometer for detecting gravitational waves. Our design envisages a ground-based tracking station in communication with two geosynchronous satellites. We assumed linearized gravitational waves in a Schwarzschild spacetime geometry outside the Earth. Our initial calculations show that such a design is sufficiently sensitive to successfully detect gravitational waves near Earth.
\end{abstract}

\section{INTRODUCTION}

When Einstein developed special relativity, the separate parameters of space and time became inextricably intertwined. The resulting concept of spacetime was further developed when Einstein understood the key to general relativity: gravity is the manifestation of curvature in spacetime. One result of this conceptualization is the existence of gravity waves, propagating ripples of spacetime caused by matter in non-spherical, non-uniform motion. Indirect experimental evidence for their existence has been observed in careful astronomical measurements of a binary pulsar. No direct detection of a gravity wave has been confirmed, but some projects with this goal are underway.

The weak coupling to matter of gravity waves makes direct detection challenging. One potential method is to use interferometers (see Figure 1). A laser, in red, is shot from $L$ to a beam splitter, $B$, where it travels to the mirrors that are acting as test masses, M, at the end of each arm. The reflected beams are then recombined

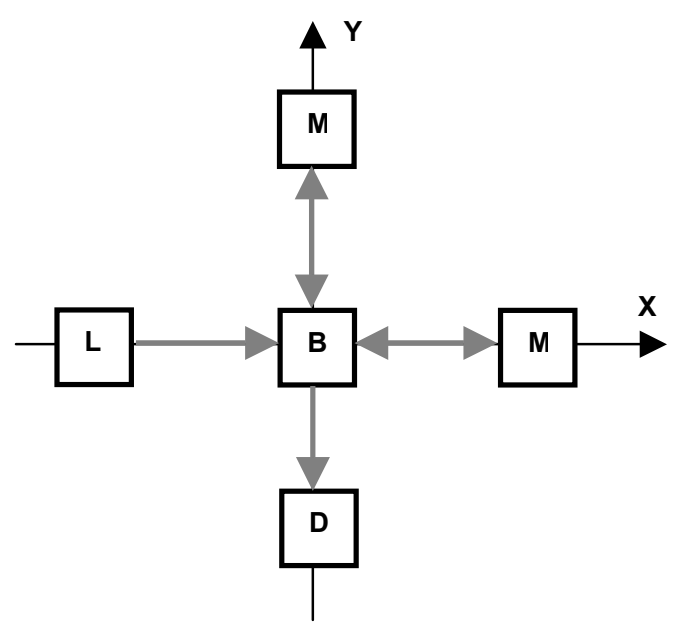

Figure 1. A schematic of an interferometer.

and because of a property of light, when light waves are combined the sum of the light waves is the result. The intensity of the resulting light wave can be measured by the detector, $D$. The conditions for constructive and destructive interference are, respectively,

$$
\Delta L \equiv L_{x}-L_{Y}=n \lambda
$$




$$
\Delta L \equiv L_{x}-L_{Y}=(n+1 / 2) \lambda
$$

for $\mathrm{n}=0,1,2 \ldots$ and light wavelength $\lambda$. Since the speed of light is constant, if the arms change length the path of the light will lengthen or shorten depending on the change. This change will cause the sum of the light waves and the amount of interference to change.

In order to understand how gravity waves will affect the interferometer, it is useful to first know more about them. Metrics of geometries close to flat spacetime, $g_{\alpha \beta}$, can be written

$$
g_{\alpha \beta}(x)=\eta_{\alpha \beta}+h_{\alpha \beta}(x)
$$

where $h_{\alpha \beta}(x)$ is a metric perturbation to the flatspace metric, $\eta_{\alpha \beta}$. Gravity waves are only perturbations to flat spacetime so their forms can be well approximated using the linearized approximation to the vacuum Einstein equation [1]. The resulting linearized gravitational wave perturbation is

$$
\begin{aligned}
& h_{\alpha \beta}(x)=\left(\begin{array}{cccc}
0 & 0 & 0 & 0 \\
0 & a & b & 0 \\
0 & b & -a & 0 \\
0 & 0 & 0 & 0
\end{array}\right) e^{t \omega(z-t)} \\
& =\left(\begin{array}{cccc}
0 & 0 & 0 & 0 \\
0 & f_{+}(t-z) & f_{x}(t-z) & 0 \\
0 & f_{x}(t-z) & -f_{+}(t-z) & 0 \\
0 & 0 & 0 & 0
\end{array}\right)
\end{aligned}
$$

If arms of equal flatspace length, $L_{*}$, are lined up along the $x$-and $y$-axes in the $z=0$ plane, then the cross polarization becomes inapplicable and the plus polarization simplifies to $f_{+}(t)=a \sin (\omega t+\delta)$. If the initial time is chosen such that $\delta=0, g_{\alpha \beta}$, then can be used to determine $\Delta \mathrm{L}$ :

$$
\Delta L=L \times a \sin (\omega t)
$$

This equation shows that longer arms will cause greater changes in the interference pattern allowing the interferometer to be more sensitive.

Several projects with the intent of detecting gravity waves are in various stages of completion. An interferometer similar to the one described above called LIGO, short for Laser Interferometer Gravitational (Wave) Observatory, is currently in its initial operational stages [2]. The disadvantage of this interferometer is that the arms are relatively short, although this has been improved upon by adding partially reflecting mirrors that effectively lengthen the arms of the interferometer. Another gravitational wave observatory based on interferometric principles that solves this problem called LISA, short for Laser Interferometer Space Antenna, has been proposed for launch into space in 2011 [2]. In space, the lengths of the arms are only limited by the power of the laser. The disadvantage of this interferometer is that once it is launched, it will be very hard to reach if problems occur or it needs technological updates. The interferometer proposed in this paper was intended to solve both of these issues. This interferometer would be based on the interferometer in Figure 1, but the test masses at the ends of the arms would be satellites with mirrors in geosynchronous orbits. This allows the arms to be about 40,000 kilometers long while keeping most of the equipment on or relatively near Earth where it can be easily repaired or updated.

\section{EARTH'S SPACETIME CURVATURE}

The reason the spacetime curvature produced by Earth won't prevent the operation of the interferometer is that the paths of the lasers are equivalent. To understand this, the design of the interferometer must be more clearly defined. Let Earth be centered on the origin of a Cartesian coordinate system with the equator on the $z=0$ (see Figure 2). The point on the Earth's equator where beam splitter B lies, along with the Earth's center, defines the x-axis. Let the satellites, $S$, be placed at points in the Earth's equatorial plane that are equidistant from the beam splitter, B, such that angle SBS is a right angle. 


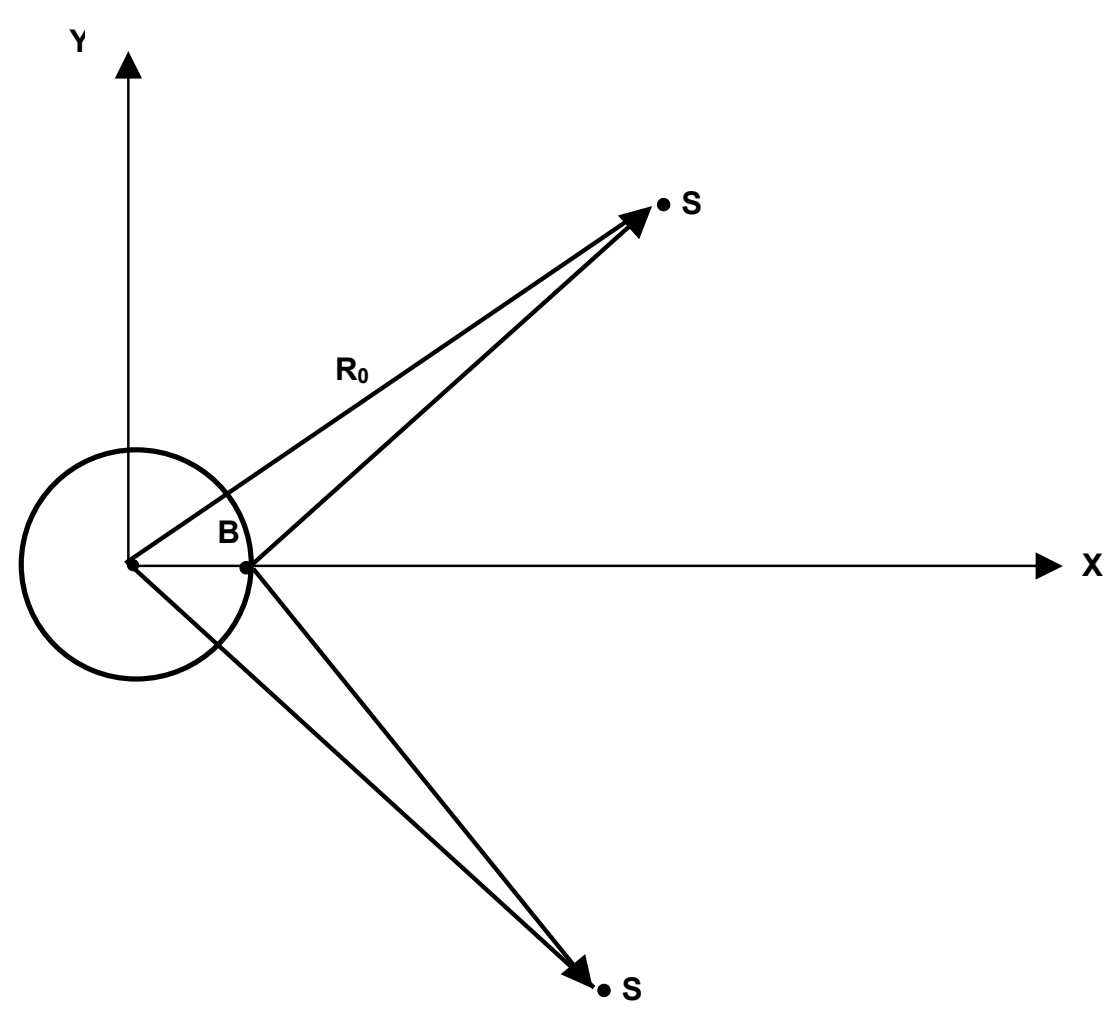

Figure 2. Two satellites (S) orbit in the equatorial plane at equal distances from an Earth-based station (B), to form an Earth-based gravity wave interferometer. $R_{0}$ is a satellite's orbital radius.

To a very good approximation, the Earth can be treated as spherically symmetric. A special metric exists for spacetime near a spherically symmetric mass $\mathrm{M}$, called the Schwarzschild metric:

$$
\begin{aligned}
& \begin{array}{llll}
t & r & \theta & \varphi
\end{array}
\end{aligned}
$$

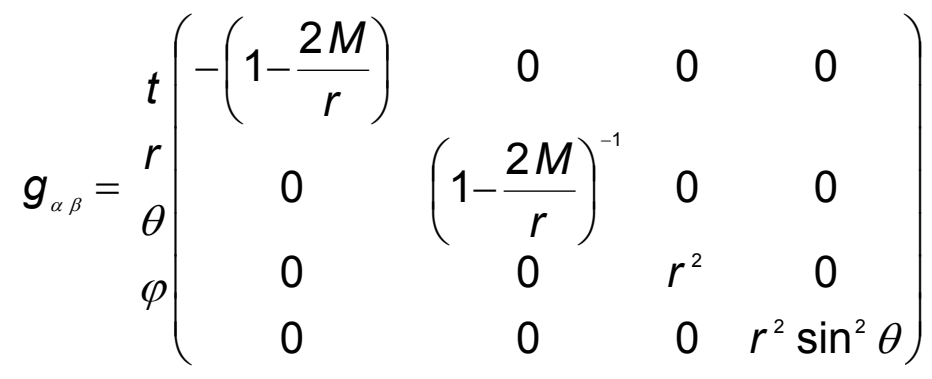

When the origin of such a coordinate system is centered on Earth, $\theta$ corresponds to latitude in radians with $\theta=0$ pointing at one pole and $\theta=\pi$ at the other, while $\varphi$ corresponds to longitude in radians with $\varphi=$ 0 pointing at the positive $x$-axis. The radius measured from the origin is $r$. Figure 2 is a cutaway of the $\theta=\pi / 2$ plane. The line element that corresponds to this metric is

$$
\begin{aligned}
d s^{2}= & -\left(1-\frac{2 M}{r}\right) d t^{2}+\left(1-\frac{2 M}{r}\right)^{-1} d r^{2} \\
& +r^{2}\left(d \theta^{2}+\sin ^{2} \theta d \varphi^{2}\right)
\end{aligned}
$$


This line element can be used to calculate distances in the Schwarzschild geometry of spacetime. If $\Delta t=\Delta \theta=\Delta \varphi=0$, this calculation simplifies to a function of the radial distance:

$$
L(r)=\int\left(1-\frac{2 M}{r}\right)^{-1 / 2} d r
$$

Since the flatspace distance from the origin to each satellite is the same, equation (8) implies the distances in Schwarzschild space must also be the same. The light reflected from the satellites also ends at the same point, the beam splitter. Because of the symmetry of this design, $\Delta \varphi$ along each arm must also be exactly the same, but it should also be clear that distance in the Schwarzschild metric is only dependant on the change in $\varphi$, not the specific values of $\varphi$. This implies the arms must be of exactly the same length and beams traveling down each arm must be traveling exactly equivalent paths. This means that the spacetime curvature produced by Earth will not effect the operation of this interferometer.

\section{LENGTH OF ARMS IN FLATSPACE}

To determine the sensitivity of the interferometer, it is necessary to find the length of the arms in flatspace. The radius of a geosynchronous orbit, $R_{0}$, must first be found by setting the centripetal force [3] acting on a satellite of mass $m$ traveling at a tangential velocity $v$ equal to the Newtonian gravitational force [3] on it due to the mass of Earth, M:

$$
\frac{m v^{2}}{R_{0}}=\frac{G M m}{\left(R_{0}\right)^{2}}
$$

Relativistic mechanics are not necessary for this calculation because space is flat and speeds involved are not significant compared to the speed of light. The satellites are geosynchronous, so they orbit Earth once per sidereal day, a day with respect to the distant stars instead of the sun. A sidereal day is about four minutes shorter than a solar day [4]. This information can be used to calculate the angular velocity of the satellite, $\omega=\pi / 43080$ radians $/ \mathrm{s}$. The tangential velocity is simply the angular velocity multiplied by the radius of orbit [3]. Substituting for $v$ into equation (9) allows the orbital radius to be solved for,

$$
R_{0}=\left(\frac{43080}{\pi}\right)^{2 / 3}(G M)^{1 / 3} \approx 4.22 \times 10^{7} \mathrm{~m}
$$

This result is obtained by letting $M=5.97 \times$ $10^{24} \mathrm{~kg}[1]$ and $\mathrm{G}=6.6726 \times 10^{-11} \mathrm{~N} \cdot \mathrm{m}^{2} / \mathrm{kg}^{2}$ [3].

Now the length of the arms can be calculated geometrically by choosing the origin of a Cartesian coordinate system to be at the beam splitter with the arms along the $x$ - and $y$-axes in the $z=0$ plane (see Figure 3).

Let vector $\mathbf{c}=\left(c_{X}, c_{Y}, 0\right)$ point from the beam splitter to the center of Earth. The lengths of the arms were defined to be equal, so let constant $k$ be this length so that $a=(k, 0,0)$ and $\mathbf{b}=(0, k, 0)$ represent the arms. The distances

$$
\begin{gathered}
R_{0}=|a-c|=\sqrt{\left(k-c_{x}\right)^{2}+\left(0-c_{y}\right)^{2}} \\
R_{0}=|b-c|=\sqrt{\left(0-c_{x}\right)^{2}+\left(k-c_{Y}\right)^{2}}
\end{gathered}
$$

are of course equal. Setting these equations equal to each other and simplifying gives the result $c_{X}=c_{Y}$. These components can be calculated by setting the equatorial radius of Earth, $R$, equal to $|\mathbf{c}|$ and solving:

$$
R=\sqrt{\left(c_{X}\right)^{2}+\left(c_{y}\right)^{2}}=\sqrt{2\left(c_{x}\right)^{2}} .
$$

Solving equation (13) we get $c_{x}= \pm R / \sqrt{ } 2$. Comparing this to Figure 3 and letting $R=$ $6.378 \times 10^{6} \mathrm{~m}[1]$, it is clear that

$$
c_{X}=c_{y}=-4.510 \times 10^{7} \mathrm{~m} .
$$

Substituting this result into either equation (11) or equation (12) and solving for $k$ results in two solutions. The negative solution can be discarded leaving

$$
\begin{gathered}
k=-\frac{R}{\sqrt{2}}+R_{0} \sqrt{1-\frac{R^{2}}{2\left(R_{0}\right)^{2}}} \\
\approx 3.74 \times 10^{7} \mathrm{~m} .
\end{gathered}
$$




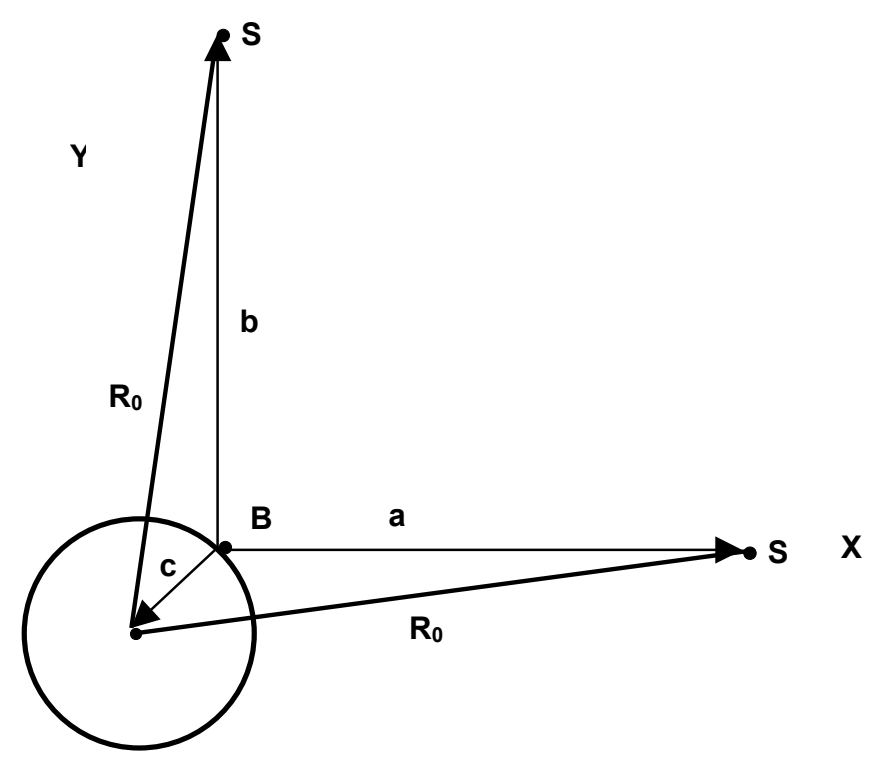

Figure 3. Geometry of an Earth-based gravity wave interferometer, with the coordinate axes rotated and shifted (from Figure 2) such that the origin is now at the beam splitter, B.

IV. LENGTHS OF ARMS IN SCHWARZSCHILD GEOMETRY

To see how the mass of Earth affects the lengths of the arms, these lengths will be calculated in the Schwarzschild geometry. This calculation will be eased by allowing the center of a Cartesian coordinate system to coincide with the center of Earth and with one of the satellites on the $x$-axis, as shown in Figure 4. Then let vector $\mathbf{B}=-\mathbf{c}$, of magnitude $R$, point from the origin to the beam splitter and vector $\mathbf{S}$ of magnitude $R_{0}$ points from the origin to the satellite on the $\mathrm{x}$-axis. Another useful tool in this calculation is a line of the form $y=m x+b$ that describes the straight path through beam splitter and the satellite on the x-axis. For reference, the parameter for the flat space lengths of the arms, $k$, has been included in Figure 4.

It has already been noted that the laser paths are identical in the Schwarzschild coordinates, so finding the new value for length $k$ in this geometry of the arm near the $x$-axis will be sufficient for both. Since $d t=d \theta=0$, the Schwarzschild line element in equation (7) simplifies to

$$
d s^{2}=\left(1-\frac{2 G M}{c^{2} r}\right)^{-1} d r^{2}+r^{2}\left(\sin ^{2} \theta d \varphi^{2}\right)
$$

where the right-hand side is now written in $\mathrm{G}$ $\neq \mathrm{C} \neq 1$ units, as will be used from here on. If $r$ is the radial position of a point along arm length $k$, then we note that $R \leq r \leq R_{0}$ implies

$$
2.10 \times 10^{-10} \leq \frac{2 G M}{c^{2} r} \leq 1.39 \times 10^{-9}
$$

a quantity much, much less than 1 . This means the right-hand side of equation (15) can be linearly approximated in $2 \mathrm{GM} /\left(\mathrm{c}^{2} \mathrm{r}\right)$

$$
d s^{2} \approx\left(1+\frac{2 G M}{c^{2} r}\right) d r^{2}+r^{2}\left(\sin ^{2} \theta d \varphi^{2}\right)
$$

Taking the square root of equation (17) and rearranging gives the result,

$$
d s \approx \sqrt{\left(1+\frac{2 G M}{c^{2} r}\right)\left(\frac{d r}{d \varphi}\right)+r^{2}} .
$$

Although this is not immediately solvable, if the line of the beam can be parameterized in terms of $r$ and $\varphi$ then it will be possible to integrate the equation.

Before the line can be written in spatial spherical polar coordinates, it must be found in spatial Cartesian coordinates. Point $\left(R_{0}, 0,0\right)$ on the line is the position of a satellite. The coordinates of another point 


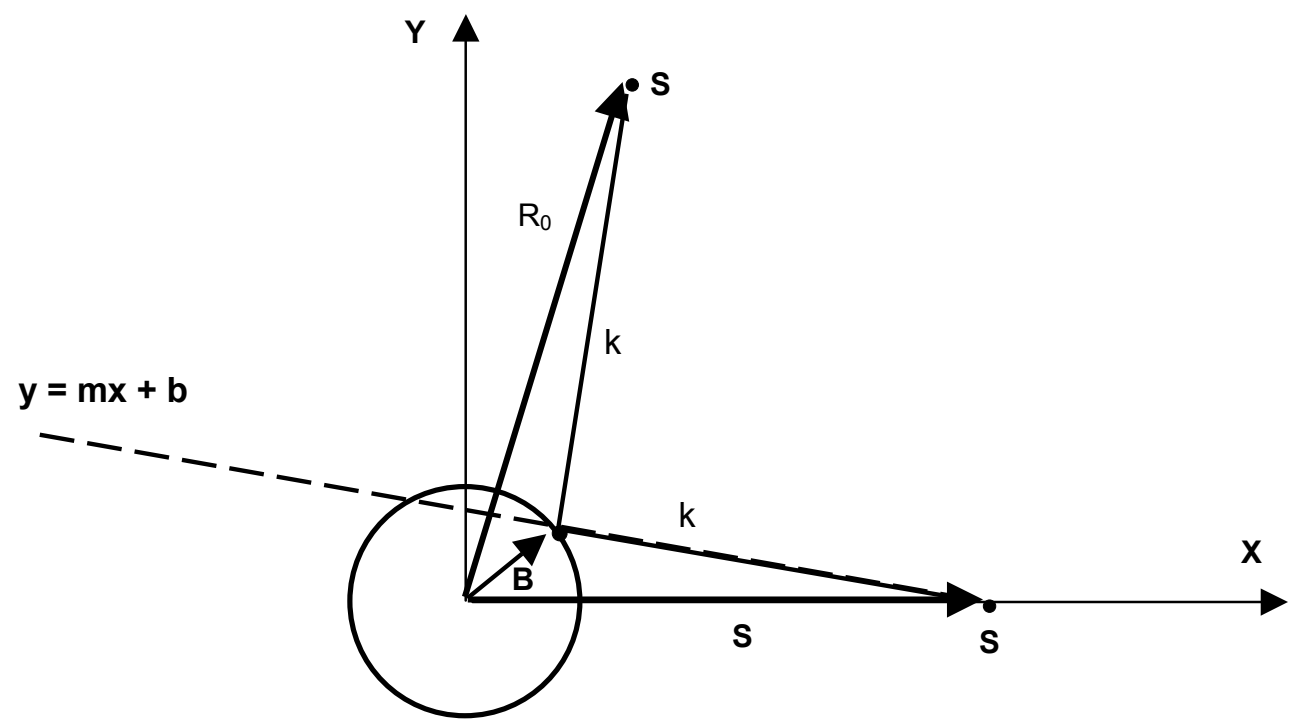

Figure 4. The layout of the gravity wave interferometer, with the coordinate origin at the Earth's center; the distance between the beam splitter and each satellite, $k$, is calculated for the Schwarzschild geometry in section IV.

correspond to the components of $\mathbf{B}=\left(\mathrm{B}_{X}\right.$, $\left.B_{Y}, 0\right)$. Since the magnitude of this vector is known,

$$
R=|B|=\sqrt{\left(B_{X}\right)^{2}+\left(B_{Y}\right)^{2}}
$$

$B_{Y}$ can be written in terms of $B_{X}$,

$$
\left(B_{y}\right)^{2}=R^{2}+\left(B_{x}\right)^{2}
$$

It is also clear that

$$
k=|S-B|=\sqrt{\left(R_{0}-B_{X}\right)^{2}+\left(B_{Y}\right)^{2}} .
$$

Substituting equation (20) into this equation and solving for $\mathrm{B}_{\mathrm{X}}$ results in

$B_{x}=\frac{\left(R_{0}\right)^{2}+R^{2}-k^{2}}{2 R_{0}} \approx 5.01 \times 10^{6} \mathrm{~m}$.

Substituting this result into equation (20), solving for $\mathrm{B}_{\mathrm{Y}}$, and choosing the positive solution gives the result

$B_{Y}=\sqrt{R^{2}+\left(B_{x}\right)^{2}} \approx 3.95 \times 10^{6} \mathrm{~m}$.

Since the coordinates of the beam splitter and the satellite are now known, the slope of the line that they are on, $m$, can be calculated:

$$
m=\frac{0-B_{y}}{R_{0}-B_{x}} \approx-0.106
$$

We also know that $0=y\left(R_{0}\right)=m \cdot R_{0}+b$, so the $y$-intercept, $b$, can be solved for:

$$
b=-m R_{0} \approx 4.47 \times 10^{6} \mathrm{~m} .
$$

If we recall the coordinate transformations from spherical polar to Cartesian coordinates,

$$
\begin{aligned}
& X=r \cos \varphi \\
& Y=r \sin \varphi
\end{aligned}
$$

The line can be written in spatial spherical polar coordinates by substituting the coordinate transformations into the equation of the line. Solving this equation for $r$ and taking the derivative with respect to $\varphi$ gives the results:

$$
\begin{gathered}
r=\frac{b}{\sin \varphi-m \cos \varphi} \\
\frac{d r}{d \varphi}=\frac{b(\cos \varphi+m \sin \varphi)}{(\sin \varphi-m \cos \varphi)}
\end{gathered}
$$


These results can be substituted into equation (18). Algebraically manipulating this result and integrating it over the values $0 \leq \varphi \leq \mathrm{B}_{\varphi}$, where $\mathrm{B}_{\varphi}$ is the $\varphi$ coordinate of the beam splitter, gives a formula for the length of the arm in the Schwarzschild geometry, $L_{s}$ :

$$
L_{s} \approx \int_{0}^{B_{\varphi}} \frac{b}{\sin \varphi-m \cos \varphi} \sqrt{1+\left(\frac{\cos \varphi+m \sin \varphi}{m \cos \varphi-\sin \varphi}\right)^{2}\left(1+\frac{2 G M(\sin \varphi-m \cos \varphi)}{c^{2} b}\right)} d \varphi
$$

The coordinate $\mathrm{B}_{\phi}$ can be found by dividing equation (27) by equation (26), solving for $\varphi$, and transforming the spatial Cartesian coordinates of the beam splitter to obtain

$$
B_{\varphi}=\tan ^{-1}\left(\frac{B_{Y}}{B_{X}}\right) \approx 0.668 \text { radians. }
$$

Equation (30) is not easily solvable by analytic techniques. It is easier to numerically solve when it is separated into a term that describes the flatspace length and one that describes the additional length caused by spacetime curvature due to the Earth's mass. The second term can be identified by the presence of the gravitational constant. This only appears in one term in equation (30), so if the equation could be separated into a part with $G$ and a part without it, the term without $G$ would represent the flatspace length of the arm, k, and the second would be the additional length due to spacetime curvature. This can be done if the equation is rearranged into the form

$$
L_{s} \approx \int_{0}^{B_{o}} \frac{b \sqrt{1+q}}{\sin \varphi-m \cos \varphi} \sqrt{1+\left(\frac{\cos \varphi+m \sin \varphi}{m \cos \varphi-\sin \varphi}\right)^{2}} d \varphi
$$

where

$$
q=\frac{2 G M(\sin \varphi-m \cos \varphi)\left(\frac{\cos \varphi+m \sin \varphi}{m \cos \varphi-\sin \varphi}\right)^{2}}{c^{2} b\left(1+\left(\frac{\cos \varphi+m \sin \varphi}{m \cos \varphi-\sin \varphi}\right)\right)}
$$

The limits $0 \leq \phi \leq \mathrm{B}_{\phi}=0.668$ radians imply $2.08 \times 10^{-10} \leq \mathrm{q} \leq 7.12 \times 10^{-10} \ll 1$, so the binomial approximation, $(1+q)^{1 / 2} \approx 1+1 / 2 q$, would be accurate [5]. Substituting this approximation into equation (32), separating the result into two terms, and letting the term without $G$ be equal to $k$ because it is the flatspace term gives the result

$$
\Delta L_{s}=L_{s}-k \approx \int_{0}^{B_{0}} \frac{G M\left(\frac{\cos \varphi+m \sin \varphi}{m \cos \varphi-\sin \varphi}\right)^{2}}{c^{2}\left(1+\left(\frac{\cos \varphi+m \sin \varphi}{m \cos \varphi-\sin \varphi}\right)^{2}\right.} \sqrt{1+\left(\frac{\cos \varphi+m \sin \varphi}{m \cos \varphi-\sin \varphi}\right)} d \varphi .
$$

This equation is manageable by a computer, so it was programmed into the software, $M A T L A B$, and solved numerically using the "quadl" command [6] with the result $\Delta \mathrm{L}_{\mathrm{S}} \approx$
$0.00442 \mathrm{~m} .<\mathrm{k}$. The length of the arm in the Schwarzschild geometry was then calculated to be $\mathrm{L}_{\mathrm{z}} \approx \Delta \mathrm{L}_{\mathrm{z}}+\mathrm{k} \approx \mathrm{k}=3.74 \times$ $10^{7} \mathrm{~m}$. 


\section{SENSITIVITY OF THE INTERFEROMETER}

To find out if the interferometer can detect gravity waves, its sensitivity must be calculated. It has been shown the spacetime curvature caused by Earth is insignificant in the lengths of the arms. Another consideration is what affect the rotation of Earth would have on this length. It can be shown rotation's effects on curvature are smaller than those found using the Schwarzschild metric, so these effects can be ignored. This means the spacetime curvature near Earth is small enough that spacetime can be approximated to be flat. Thus the gravitational wave equations already calculated in section four are accurate in this situation.

To calculate the sensitivity of the interferometer, return to the arrangement of the interferometer with respect to the coordinate system in Figure 1. Assume that this is the $z=0$ plane and that a gravity wave is propagating in the z-direction. Choose the initial time, $\mathrm{t}=0$, such that $\delta=0$ and let the flatspace length of the arms be $L$ * $\approx \mathrm{k}$. Let $a \approx 10^{-21}$ since that is the expected amplitude of gravity waves that will be detectable on Earth [1]. In this situation, equation (4) can be used to calculate the maximum difference in the lengths of the arms, $\Delta \mathrm{L}_{\text {MAX }}$, when a gravity wave passes with the maximum occurring when $\sin (\omega t)=$ 1. It is more useful to use the wavelength of the laser, $\lambda$, to calculate the fraction of a wavelength the lengths of the arms will change, $f$. For a typical Helium-Neon laser, $\lambda \approx 633 \times 10^{-9} \mathrm{~m}$, the fraction is

$$
f=\frac{\Delta L_{\text {MAX }}}{\lambda}=\frac{L_{.} a}{\lambda} \approx 5.91 \times 10^{-8} \text {. }
$$

Because of the partially reflecting mirrors, the effective lengths of LIGO's arms are $L_{*} \approx$ $8 \times 10^{5} \mathrm{~m}$ [7]. Substituting this value into equation (35) gives the result $\mathrm{f}_{\mathrm{LIGO}} \approx 1.26 \times$ $10^{-9}$. It is reported [1] that the initial LIGO detector will be able to detect $\mathrm{f}_{\mathrm{LIGO}}$, reported $\approx$ $10^{-9}$, so these values are consistent. If the proposed interferometer were operating with equipment similar to LIGO's such that it was capable of detecting $f \approx 10^{-9}$, then it would be able to detect gravity waves of $a \sim 10^{-21}$. Table 1 is a partial reproduction of the table found on page 171 of Bartusiak [7] of expected rates of gravitational wave detections that LIGO will make.

\section{CONCLUSION}

The proposed interferometer can be expected to successfully make a gravitational wave detection given enough time according to the theoretical considerations made. Since it is more sensitive than LIGO, it would also make more detections than those expected as listed in Table 1 . It is also a safer expenditure than LISA because it is repairable and updatable. Thus the interferometer is at least worth further investigation.

The next step in making this interferometer a reality would be to consider the experimental issues. Many of these have already been solved in the process of creating LIGO and LISA, but it will also provide new challenges. LIGO's arms are contained in tubes that are not practical for this interferometer. On the other hand, LISA's arms will be in the vacuum of space. Interactions with Earth's atmosphere will introduce difficulties that neither of these other two interferometers encountered.

\begin{tabular}{|c|c|c|}
\hline Event & Region of Space & Detection Rate \\
\hline Supernova & Within Milky Way & 1 to 3 per century \\
\hline Black Hole/Black Hole Merger & 300 million light-years & $\begin{array}{c}1 \text { per } 1,000 \text { years } \\
\text { to } 1 \text { per year }\end{array}$ \\
\hline Neutron Star/ Neutron Star Merger & 60 million light-years & $\begin{array}{r}1 \text { per } 10,000 \text { years } \\
\text { to } 10 \text { per century }\end{array}$ \\
\hline Neutron Star/ Neutron Star Merger & 130 million light-years & $\begin{array}{r}1 \text { per } 10,000 \text { years } \\
\text { to } 10 \text { per century }\end{array}$ \\
\hline
\end{tabular}

Table 1. LIGO's Expected Detection Rates of Gravitational Wave Detections [7]. 
Solving the experimental difficulties such as this one could make this interferometer a practical solution for a gravitational wave observatory of the future.

\section{ACKNOWLEDGEMENTS}

I wish to thank Dr. C. C. Chancey, those involved in the Gravitational Wave Astronomy Summer School, and the University of Northern lowa Physics Department for their guidance, advice, and support.

\section{REFERENCES}

1. Hartle, James B. (2003). Gravity: An Introduction to Einstein's General Relativity. San Francisco, CA: Addison Wesley.

2. Hughes, Scott A. (2004, June). Listening to the Universe with Gravitational-Wave Astronomy. Paper presented at the meeting of the $1^{\text {st }}$ Gravitational Wave Astronomy Summer School, South Padre Island, TX.
3. Tipler, Paul A. (1999). Physics for Scientist and Engineers ( $4^{\text {th }}$ ed.). New York, NY: W. H. Freeman and Company.

4. Pasachoff, Jay M. and Filippenko, Alex. (2001). The Cosmos: Astronomy in the New Millenium. Pacific Grove, CA: Brooks/Cole - Thomson Learning.

5. Taylor, John R., Zafiratos, Chris R., and Dubson, Michael A. (2004). Modern Physics for Scientists and Engineers $\left(2^{\text {nd }}\right.$ ed.). Upper Saddle River, $\mathrm{NJ}$ : Prentice Hall.

6. Hanselman, Duane and Littlefield, Bruce. (2001). Mastering MATLAB 6: A Comprehensive Tutorial and Reference. Upper Saddle River, NJ: Prentice Hall.

7. Bartusiak, Marcia. (2003). Einstein's Unfinished Symphony: Listening to the Sounds of Space-Time. New York, NY: Berkley Books.

University of Northern lowa Physics Department http://www.phvsics.uni.edu

\section{University of Northern lowa Center for Energy and}

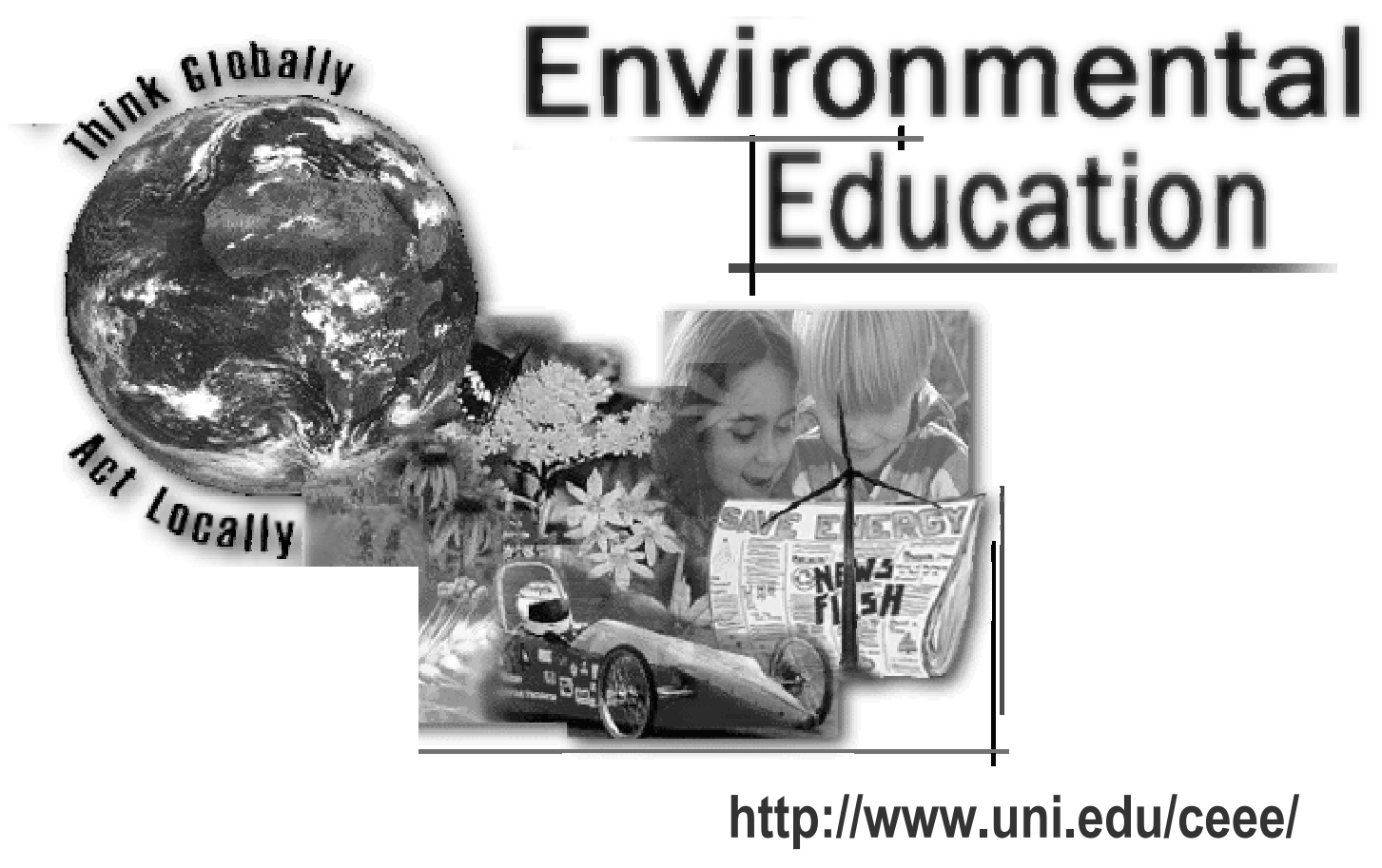


